\title{
Microwave scattering from ice crystals: how much parameters can differ from equal volume spheres
}

\author{
F. Teschl, W. L. Randeu, and R. Teschl \\ Department of Broadband Communications, Graz University of Technology, Graz, Austria \\ Received: 3 November 2009 - Revised: 19 March 2010 - Accepted: 26 March 2010 - Published: 8 June 2010
}

\begin{abstract}
The scattering properties of ice crystals at centimeter and millimeter wavelengths are fundamental for their measurement with radars or radiometers as well as for many other applications in remote sensing. Only the scattering properties of homogeneous spheres can be computed in a mathematically exact way using the Mie theory. Therefore, for many approaches ice crystals and other frozen hydrometeors are approximated by ice spheres that have the same volume as the crystal or hydrometeor - so called equal volume spheres. However, many frozen hydrometeors are nonspherical. They exist in a huge variety of shapes and exhibit different mixtures of ice, water and air. Therefore it is desirable to accurately compute scattering from non-spherical particles in order to clearly understand the effect the shape of a hydrometeor has on its scattering pattern.

In this study, single scattering parameters like scattering cross section (Csca) and absorption cross section (Cabs) were calculated for six pristine ice crystals by means of the Discrete Dipole Approximation (DDA). The calculations were carried out for frequencies from $1 \mathrm{GHz}$ to $300 \mathrm{GHz}$ - leading to size parameters of up to 4.

The study calculates the scattering parameters for randomly oriented ice crystals and investigates to what extent the exact scattering parameters can differ from an approximation by equal volume spheres.
\end{abstract}

\section{Introduction}

The detection and measuring of frozen precipitation and cloud particles becomes more and more important for climate and weather studies. Frozen precipitation represents a significant part of global precipitation and is, in the polar re-

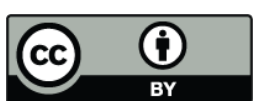

Correspondence to: $\mathrm{F}$. Teschl (franz.teschl@tugraz.at) gion, crucial for the mass of the polar caps. Ice clouds can be found at all latitudes and they have an eminent influence on the climate on our planet. Besides, they also influence the quality of satellite communication links.

When detecting frozen hydrometeors with radiometers working at millimeter wavelength (e.g. Skofronick-Jackson et al., 2004), radiative transfer models are needed in which the assumed scattering parameters are of fundamental importance. The calculation of scattering parameters of ice crystals and frozen hydrometeors is a time consuming task. Therefore for many approaches ice crystals and other frozen hydrometeors are parameterized as equal volume ice spheres and their exact scattering solution (Mie, 1908) is used. Indeed it has been reported by Kim (2006) that scattering and absorption cross sections of randomly oriented snow crystals do not depend on the specific particle shape for size parameters $<2.5$. The size parameter $x$ is defined as

$x=2 \pi a / \lambda$

where $a$ is the radius of an equal volume sphere and $\lambda$ is the wavelength of the incoming wave.

In this study, it was verified whether the rule above also holds for the regarded pristine ice crystals and how big the differences are on average. Furthermore, it was investigated to what degree the approximation by equal volume ice spheres can differ in the worst case for "extreme" crystal orientations, and if and how the situation changes from the regime with $2 \pi a / \lambda<2.5$ to that with $2 \pi a / \lambda>2.5$.

This paper is a sequel to a study presented by Teschl et al. (2009). The structure is as follows: In Sect. 2 the index of refraction of ice is shortly introduced and it is addressed what model has been adopted for the calculations. In Sect. 3 the DDA is shortly described with special emphasis on its accuracy. In Sect. 4 the DDA scattering results of reference objects are presented in order to show if the accuracy of the method is sufficient. In Sect. 5 the six pristine ice crystal shapes that have been chosen for the study are presented and

Published by Copernicus Publications on behalf of the European Geosciences Union. 
their geometry and dimensions are given. Then in Sect. 6 the scattering and absorption results for these crystals are presented and finally they are discussed and summarized in Sect. 7.

\section{Index of refraction of ice}

The index of refraction of ice is a complex number and strongly dependent on frequency and temperature. In literature several papers exist on the index of refraction of ice at microwave frequencies (e.g., Ray, 1972; Warren, 1984; Mätzler and Wegmüller, 1987). These studies differ mainly in the imaginary part of the refractive index. However, it has been shown that the different models for the index of refraction have minor influence on the scattering cross section, the backscattering cross section, and on the asymmetry factor of ice crystals at centimeter and millimeter wavelengths (Teschl et al., 2009). For the calculations in this study the model of Ray (1972) has been adopted. The temperature was chosen to be $-5^{\circ} \mathrm{C}$. (For example, the complex index of refraction $n$ of ice at $10 \mathrm{GHz}$ and $-5^{\circ} \mathrm{C}$ is $1.78-\mathrm{j} 0.0002$.)

\section{Discrete Dipole Approximation}

Scattering parameters were calculated using the Discrete Dipole Approximation (DDA). The DDA was first described by Purcell and Pennypacker (1973). It is a powerful technique for computing scattering and absorption by targets of arbitrary geometry (cf. Draine and Flatau, 1994). The DDA models the actual particle by small subunits. The subunits must be small enough compared to the wavelength so that each subunit can be represented as a single dipole. The dipoles are located on a cubic lattice. Each dipole is subjected to the electric field of the incident wave and to the electric fields of all other dipoles.

The DDA is completely flexible regarding the geometry of the particle, being limited only by the need that the separation $d$ between the dipoles is small compared to the wavelength $\lambda$ and any structural length in the particle (cf. Draine and Flatau, 2010). The accuracy of the DDA can be increased by increasing the number of dipoles. The dominant error in DDA calculations arises because of the inaccurate representation of the particle surface by the dipoles (Okamoto et al., 1995; Lemke et al., 1998).

Increasing the number of dipoles, however, implicates additional calculation time. So the number of dipoles must be chosen high enough to ensure a certain accuracy, and low enough in order not to overburden computing resources.

According to Draine and Flatau (1994) the following criterion should be satisfied for a passable accuracy of the DDA:

$|n| k d<1$

where $n$ is the complex index of refraction of the target material, $d$ is the separation between the dipoles, and $k=2 \pi / \lambda$.
However, for accurate calculations of the scattering phase function (e.g., for radar cross-sections), a more conservative criterion

$|n| k d<0.5$

is suggested (cf. Draine and Flatau, 2010).

The applied DDA software uses in its default settings a comparable criterion, namely that there should be at least 10 diploes per wavelength inside the particle. For higher indices of refraction the number of dipoles should be even higher according to

$10|n| d>\lambda$

As for ice the absolute value of the index of refraction is about 1.78 for frequencies from 1 to $300 \mathrm{GHz}$, here the scattering objects were modeled with at least 18 dipoles per wavelength.

The DDA has already been used to calculate microwave scattering from ice crystals by Evans and Stephens (1995), Lemke and Quante (1999), Kim (2006) or Hong (2007). The calculations in this study were carried out with the relatively new software package ADDA, version 0.79 (developed by Maxim A. Yurkin and Alfons G. Hoekstra at the University of Amsterdam). ADDA is available under GNU general public license (available at: http://www.science.uva.nl/research/ scs/Software/adda/, last access: 19 March 2010).

\section{Scattering parameters of reference objects}

Before using ADDA to calculate the scattering parameters of simple ice crystals, its accuracy was reviewed by modeling a sphere and comparing the ADDA results with the exact Miesolution in a frequency range from 1 to $300 \mathrm{GHz}$. Furthermore, ADDA was applied to calculate the scattering parameters of an ice cube with the same volume as the sphere. It was observed what maximum deviation of the scattering parameters of that ice cube can occur when assuming randomly oriented cubes. Since the aspect ratio of a cube is 1 , no extreme deviations were expected in contrast to ice needles or ice plates where aspect ratios of 100 are not exceptional.

Figure 1 shows the dipole assignment for the modeled ice sphere and the ice cube. The sphere has a diameter of $1 \mathrm{~mm}$ resulting in a volume of $0.524 \mathrm{~mm}^{3}$. The cube with an equivalent volume has a side length of $0.806 \mathrm{~mm}$. The sphere and the cube were modeled with a grid of 16 dipoles in the $x, y$, and $\mathrm{z}$ axis. The sphere was modeled by 2176 single dipoles, for the cube all $4096\left(=16^{3}\right)$ dipoles were assigned. For the sphere, a volume correction method was used. Therefore it was assured that the volume of the dipole representation is exactly the same as the geometrical sphere, accepting that the diameter of the dipole representation slightly differs from the nominal. In the present case the diameter of the dipole representation is $0.9952 \mathrm{~mm}$ instead of 1 . 

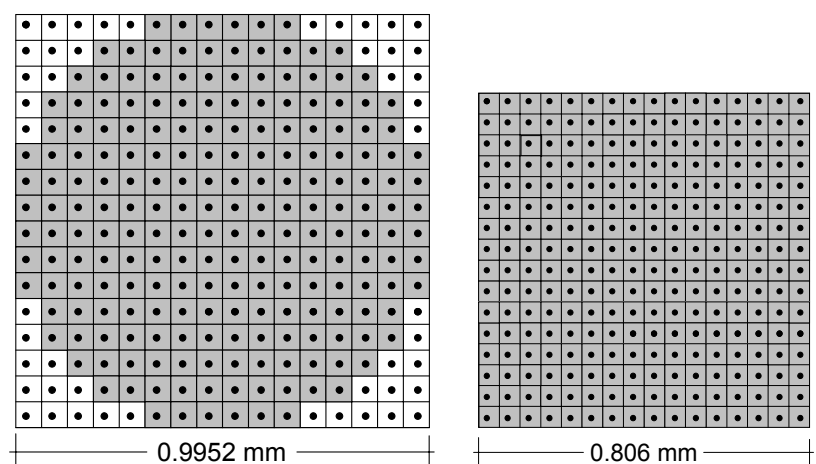

Fig. 1. Dipole assignment for the modeled ice sphere and ice cube (2-D projection). Assigned dipoles are gray and void dipoles are white. For the sphere 2176 dipoles and for the cube 4096 dipoles were assigned. The volume of both objects is that of a geometric sphere with $1 \mathrm{~mm}$ in diameter, namely $0.524 \mathrm{~mm}^{3}$.

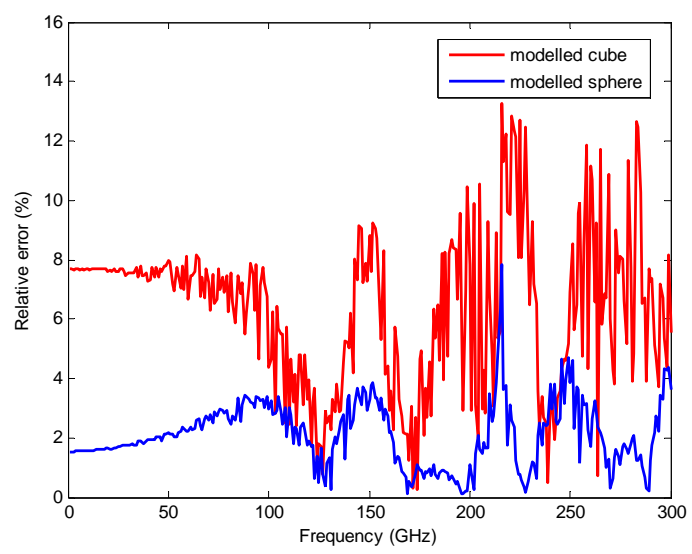

Fig. 2. Maximum relative error of Csca of randomly oriented ice cubes and ice spheres as a function of the frequency compared to the exact Mie solution of an equal volume sphere.

The scattering cross section (Csca) and the absorption cross section (Cabs) were calculated for the modeled ice sphere and ice cube for 1 to $300 \mathrm{GHz}$ in $1 \mathrm{GHz}$ steps. For the scattering calculation both objects where randomly oriented. For each frequency two random orientations were calculated and the maximum deviation from the Mie-solution was determined.

Figure 2 shows the maximum relative error of Csca. It can be seen that the error of the randomly oriented cube is about three times as large on average as that of the randomly oriented sphere model. The average deviation over the whole frequency range is $6 \%$ for the modeled cube and $2 \%$ for the modeled sphere. The maximum deviation of the ice cube is $13 \%$ - the maximum deviation of the DDA modeled sphere is $8 \%$. Figure 2 also shows that the deviation does not necessarily increase with increasing frequency (increasing size parameter).
Table 1. Size parameter and number of dipoles per wavelength for the modeled sphere and cube for selected frequencies/wavelengths.

\begin{tabular}{cccrr}
\hline $\begin{array}{c}\text { Frequency } \\
(\mathrm{GHz})\end{array}$ & $\begin{array}{c}\text { Wavelength } \\
(\mathrm{mm})\end{array}$ & $\begin{array}{c}\text { Size } \\
\text { parameter }\end{array}$ & $\begin{array}{c}\text { Dipoles per wavelength } \\
\text { Sphere }\end{array}$ & \begin{tabular}{c} 
Cube \\
\hline 1
\end{tabular} \\
300 & 0.01 & 4823.15 & 5955.36 \\
30 & 10 & 0.31 & 160.77 & 198.51 \\
100 & 3 & 1.05 & 48.23 & 59.55 \\
150 & 2 & 1.57 & 32.15 & 39.70 \\
200 & 1.5 & 2.09 & 24.12 & 29.78 \\
250 & 1.2 & 2.62 & 19.29 & 23.82 \\
300 & 1 & 3.14 & $* 18.09$ & 19.85 \\
\hline
\end{tabular}

* Dipole grid was increased to $18 \times 18 \times 18$ in order to fulfill criterion (4).

The number of dipoles per wavelength in the dipole models vary from several thousands at low $\mathrm{GHz}$ frequencies to about 20 at $300 \mathrm{GHz}$ (Table 1). Criterion (4) for applicability of the DDA is therefore always fulfilled. For low frequencies the number of dipoles is even 100 times more than required. For frequencies up to about $30 \mathrm{GHz}$ the plotted deviations compared to the exact Mie solution are quite smooth. At frequencies higher than $30 \mathrm{GHz}$ they start to become noisy. For the regarded sphere with diameter $D=1 \mathrm{~mm}$, $30 \mathrm{GHz}(\lambda=10 \mathrm{~mm})$ is practically the frequency up to which the Mie theory can be reduced to the Rayleigh approximation $(D<\lambda / 10)$. The calculations were carried out with randomly oriented particles. The results support that the orientation of the particle has no influence on the scattering parameters for wavelengths longer than 10 times the particle diameter. However, within this frequency range the scattering parameters of the modelled ice sphere and ice cube differ uniformly by a few percent although their volume is exactly the same.

Figure 3 shows the maximum relative error of Cabs. Again the error of the randomly oriented cube is on average three times as large as that of the randomly oriented sphere model. The average deviation over the whole frequency range is $9 \%$ for the modeled cube and 3\% for the modeled sphere. The maximum deviation of the ice cube is $26 \%$ - the maximum deviation of the DDA modeled sphere is $13 \%$. Again for frequencies up to $30 \mathrm{GHz}$ the error is mainly stable even for arbitrarily oriented objects.

The results of the ice sphere show that the used number of dipoles per wavelength is sufficient to keep the error within a few percent. For the modeling of the ice crystals therefore an equivalent dipole grid was used. 


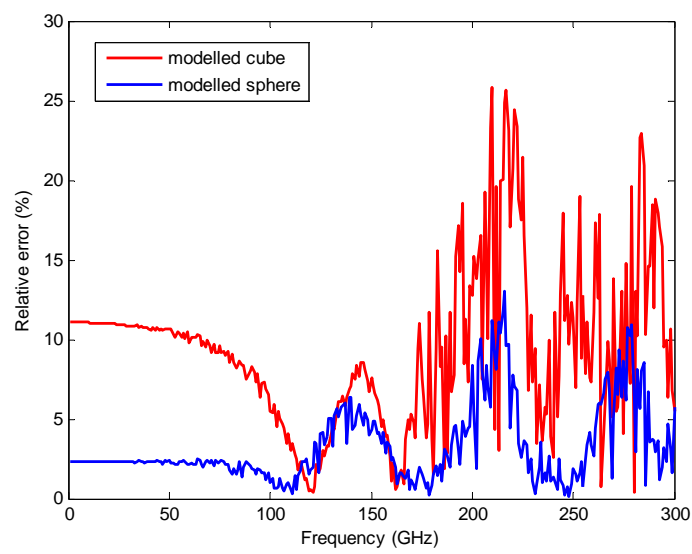

Fig. 3. Maximum relative error of Cabs of randomly oriented ice cubes and ice spheres as a function of the frequency compared to the exact Mie solution of an equal volume sphere.

\section{Ice crystal models}

The scattering parameters of pristine ice crystals have been calculated and it was observed how these parameters can maximally differ from those of equal volume ice spheres for special crystal orientations. The ice crystals are simple columns, plates and dendrites - the crystal types C1e, C1g, P1a, P1c, and P1d of the classification of Magono and Lee (1966). Ice crystal shapes obey certain dimensional relationships. Thickness and diameter of plate-like crystals and length and width of columnar crystals are characteristically related to each other. Table 2 shows these relationships for the modeled crystal shapes. Table 2 also gives a range for the sizes occurring in nature. For the ice crystal dipole models these dimensional relationships were applied and in each case the maximum possible size was assumed. The crystals were assumed to consist of solid ice and the index of refraction according to Ray $(1972)$ at $-5{ }^{\circ} \mathrm{C}$ was applied. The equal volume sphere radii of the modeled crystals are listed in Table 3.

The separation $d$ between the dipole in the crystal models at least comply with Criterion (4). The calculations were carried out in $1 \mathrm{GHz}$ steps up to a frequency of $300 \mathrm{GHz}$. For each frequency two random orientations of the crystal were calculated.

\section{Scattering and absorption results}

The determined scattering cross sections of the six ice crystal shapes are presented in Fig. 4 on a logarithmic scale. The different crystal results are shown in different colors. The solid lines give in each case the scattering cross section of an equal volume sphere. The dots represent results of arbitrarily oriented ice crystals. The Csca is as a rule the higher, the higher the volume of the crystal is. The results of the arbitrarily oriented ice crystals are spread around the equal volume sphere
Table 2. Dimensional relationships for the modeled ice crystal types (according to Pruppacher and Klett, 1978). The crystal code refers to the classification of Magono and Lee (1966); Length (L), height (h), and diameter of the circle circumscribed around the crystal (d).

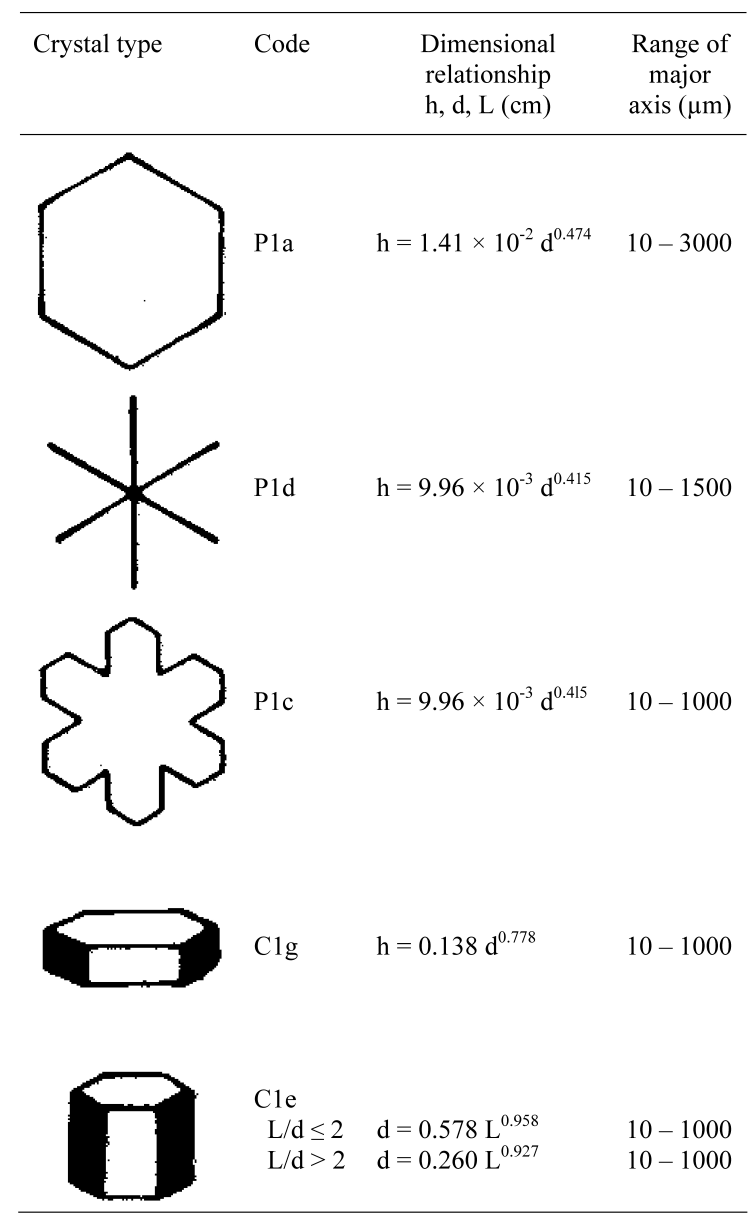

Table 3. Equal volume sphere radius of the modeled ice crystals and size parameter at $300 \mathrm{GHz}$ frequency.

\begin{tabular}{lcc}
\hline $\begin{array}{l}\text { Crystal } \\
\text { Code }\end{array}$ & $\begin{array}{c}\text { Equi volume sphere } \\
\text { radius }(\mathrm{mm})\end{array}$ & $\begin{array}{c}\text { Size parameter } \\
\text { @ 300 GHz }\end{array}$ \\
\hline P1a & 0.64 & 4.03 \\
P1d & 0.18 & 1.16 \\
P1c & 0.30 & 1.88 \\
C1g & 0.34 & 2.13 \\
C1e-2 $(\mathrm{L} / \mathrm{d} \leq 2)$ & 0.42 & 2.66 \\
C1e-1 $(\mathrm{L} / \mathrm{d}>2)$ & 0.26 & 1.64 \\
\hline
\end{tabular}

curves. The relative deviation from the sphere can be $100 \%$ and more. Nevertheless for a given frequency the possible Csca values of the individual ice crystals overlap by a small degree only. 


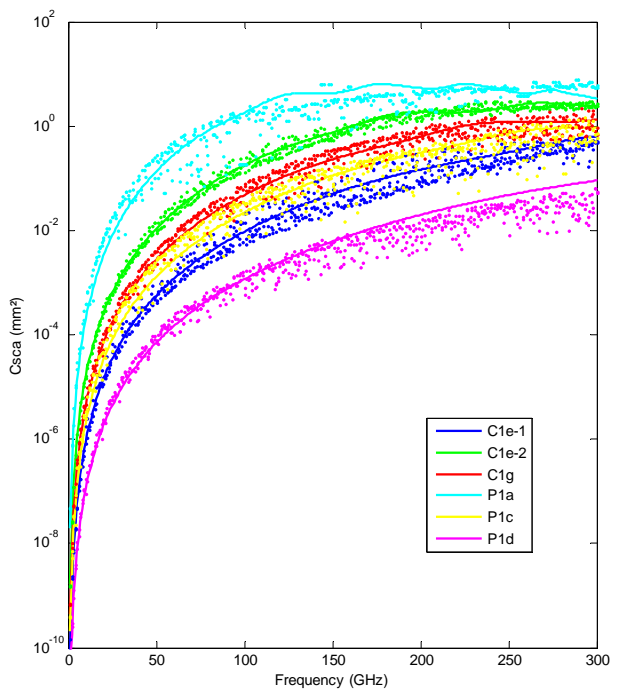

Fig. 4. Csca of arbitrary oriented pristine ice crystals as a function of the frequency. As a reference in each case the curve of an equal volume sphere is given.

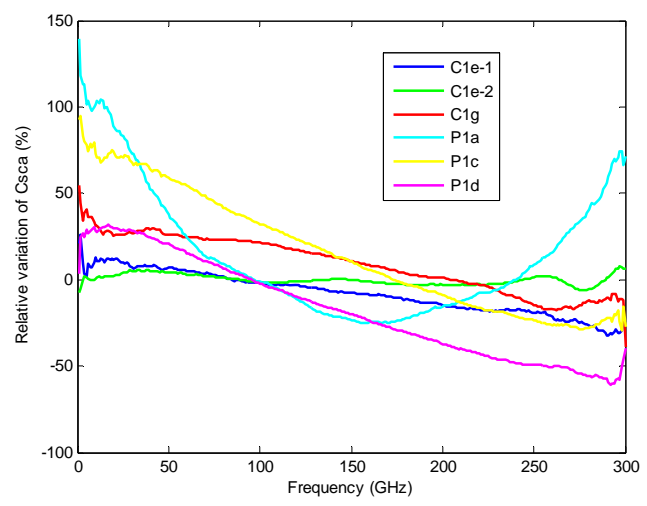

Fig. 5. Relative variation of Csca of ice crystals (averaged orientation) compared to an equal volume sphere.

Figure 5 shows the variation of Csca for averaged orientation of the ice crystals relative to the equal volume sphere. Positive values indicate that the Csca of the ice crystal is higher than that of its equal volume sphere. The curves show a trend that for the observed ice crystals the variation decreases with increasing frequency and mostly becomes negative at $300 \mathrm{GHz}$. It is also noticeable that the curves of crystals with moderate aspect ratios as $\mathrm{Cle}$ or $\mathrm{C} 1 \mathrm{~g}$ are rather flat and don't exhibit such a strong variation as very elongated crystals with extreme aspect ratios.

The maximum difference between the Csca of arbitrarily oriented ice crystal and that from equal volume ice spheres is plotted in Fig. 6. For the regarded ice crystals Csca of arbitrarily oriented particles can be more than twice as high or less than half of that of the equal volume ice sphere in the plotted frequency range. The lowest deviations from the

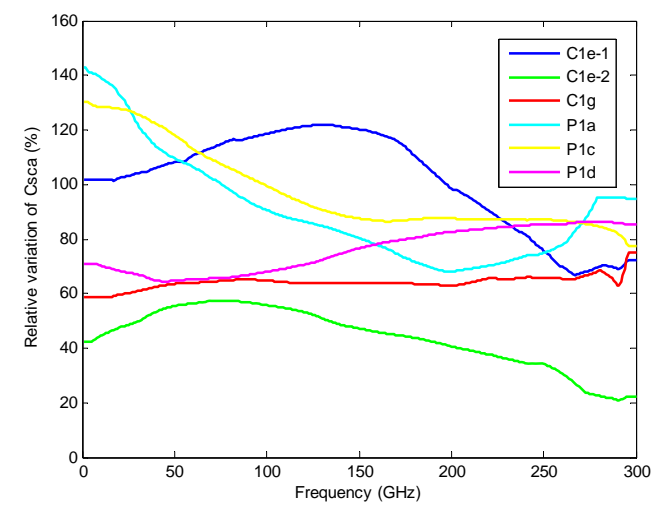

Fig. 6. Maximum relative variation of Csca of ice crystals compared to an equal volume sphere.

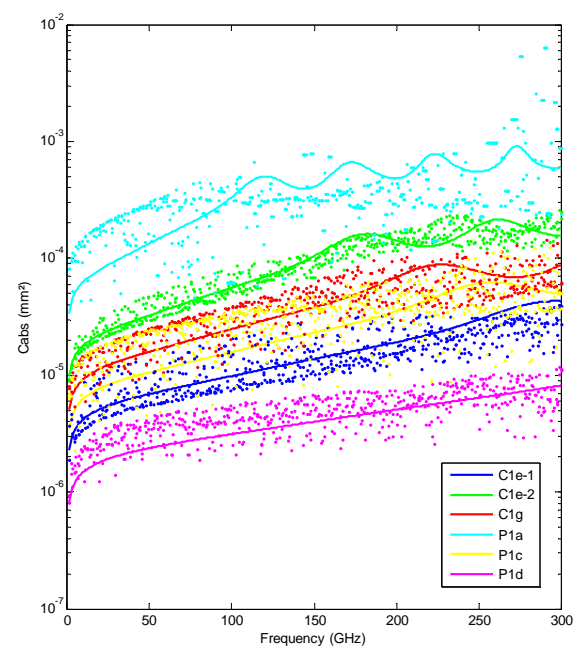

Fig. 7. Cabs of arbitrarily oriented pristine ice crystals as a function of the frequency. As a reference in each case the curve of an equal volume sphere is given.

sphere have been observed for the crystal type Cle-2 which has an aspect ratio close to 1 .

The absorption cross section (Cabs) calculation of the six ice crystals are presented on a logarithmic scale in Fig. 7. Again the solid lines give in each case the absorption cross section of an equal volume sphere - the dots represent results of arbitrarily oriented ice crystals. The variation of Cabs compared to the equal volume sphere shows a behavior comparable to that of Csca for the individual crystal types. Again the variation mostly decreases with increasing frequency when averaging over all orientations (Fig. 8). The maximum observed deviation of Cabs is also comparable to that of Csca (Fig. 9). For the crystal with the largest volume (P1a) the maximal deviation increases rapidly at $200 \mathrm{GHz}$ (size parameter $x=2.7$ ) and above. 


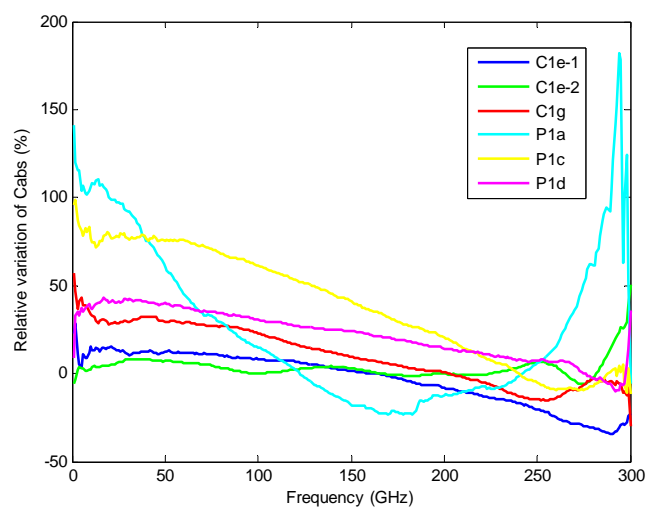

Fig. 8. Relative variation of Cabs of ice crystals (averaged orientation) compared to an equal volume sphere.

\section{Discussion and conclusion}

For the calculated frequency range the total number of dipoles was held fixed. On the one hand this approach ensures that the modeled particle has the same "faceted" shape at all frequencies. On the other hand this implicates that at the lower end of the frequency range the number of dipoles per wavelength is very high while at the higher end of the frequency range the number simply fulfills the requirements. However, holding the number of dipoles per wavelength fixed is not practicable for this wide frequency range. Using the minimum of 18 dipoles per wavelength, would "model" realistic ice crystals at centimeter wavelengths by one dipole only. A higher number of dipoles that would allow recognizing the shape at centimeter wavelengths would overburden computing resources at millimeter wavelengths.

In relevant literature it has been reported that scattering and absorption cross sections of randomly oriented idealized snow crystals do not depend on the specific particle shape for size parameters $x<2.5$. In the present study scattering parameters of simple ice crystals have been calculated up to $300 \mathrm{GHz}$ leading to size parameters of up to 4 . The present results do not affirm that a size parameter of 2.5 represents a threshold. Indeed the deviation of parameters of the crystal P1a that reaches a size parameter of 4 at $300 \mathrm{GHz}$ increase at about $200 \mathrm{GHz}$. But it has been found that at low size parameters or frequencies also high deviations (to equal volume spheres) occur. Since the ice crystals have been modeled with a dipole grid of $16 \times 16 \times 16$ especially at low frequencies the number of dipoles is 100 times more than required for DDA modeling. The results at low frequencies are therefore even more trustable.

The results of this study also show that the scattering parameters of ice crystals can vary up to $150 \%$ and more from those of equal volume ice spheres for frequencies up to $300 \mathrm{GHz}$ or size parameters up to 4 . It is further observed that the crystals with extreme aspect ratios (extremely flat or long) show higher maximum deviations than those with moderate aspect ratios.

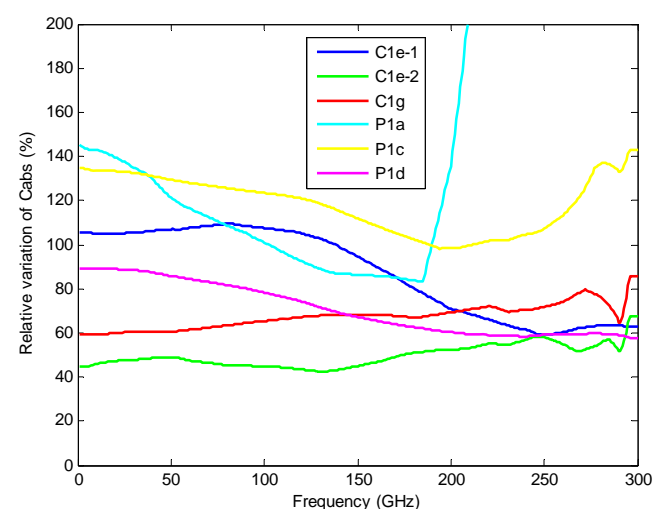

Fig. 9. Maximum relative variation of Cabs of ice crystals compared to an equal volume sphere.

Acknowledgements. The authors thank Maxim A. Yurkin and Alfons G. Hoekstra from the University of Amsterdam for providing the ADDA code and two anonymous reviewers for valuable comments.

Edited by: S. C. Michaelides

Reviewed by: two anonymous referees

\section{References}

Evans, K. F. and Stephens, G. L.: Microwave Radiative Transfer through Clouds Composed of Realistically Shaped Ice Crystals, Part I, Single Scattering Properties, J. Atmos. Sci., 52, 20412057, 1995.

Draine, B. T. and Flatau, P. J.: Discrete-dipole approximation for scattering calculations, L. Opt. Soc. Am., A11, 1491-1499, 1994.

Draine, B. T. and Flatau, P. J.: User Guide to the Discrete Dipole Approximation Code DDSCAT 7.1, available at: http://arXiv. org/abs/1002.1505v1, last access: 19 March 2010, 2010.

Hong, G.: Parameterization of scattering and absorption properties of nonspherical ice crystals at microwave frequencies, J. Geophys. Res., 112, D11208, doi:10.1029/2006JD008364, 2007.

Kim, M.-J.: Single scattering parameters of randomly oriented snow particles at microwave frequencies, J. Geophys. Res., 111, doi:10.1029/2005JD006892, 2006.

Lemke, H. and Quante, M.: Backscatter characteristics of nonspherical ice crystals: Assessing the potential of polarimetric radar measurements, J. Geophys. Res., 104(D24), 31739-31752, 1999.

Lemke, H., Okamoto, H., and Quante, M.: Discussion: Comment on error analysis of backscatter from discrete dipole approximation for different ice particle shapes [Liu, C.-L., Illingworth, A. J., 1997, Atmos. Res. 44, 231-241.], Atmos. Res., 49, 189-197, 1998.

Magono, C. and Lee, C.W.: Meteorological classification of natural snow crystals, Journal of the Faculty of Science, Hokkaido University, Series 7, Geophysics, 2, 321-335, 1966.

Mätzler, C. and Wegmüller, U.: Dielectric Properties of freshwater ice at microwave frequencies, J. Phys. D: Appl. Phys., 20, 16231630, 1987 [Erratum: J. Phys. D: Appl. Phys., 21, 1660, 1988]. 
Mie, G.: Beiträge zur Optik trüber Medien, speziell kolloidaler Metallösungen, Annalen der Physik, Vierte Folge, 25, 377-445, 1908.

Okamoto, H., Macke, A., Quante, M., and Raschke, E.: Modeling of backscattering by non-spherical ice particles for the interpretation of cloud radar signals at $94 \mathrm{GHz}$. An error analysis, Contrib. Atmos. Phys., 68, 319-334, 1995.

Pruppacher, H. R. and Klett, J. D.: Microphysics of Clouds and Precipitation, D. Reidel Publishing Company, Dortrecht, The Netherlands, 1978.

Purcell, E. M. and Pennypacker, C. R.: Scattering and absorption of light by nonspherical dielectric grains, Astrophys. J., 186, 705714, 1973.
Ray, P.: Broadband complex refractive indices of ice and water, Appl. Opt., 11, 1836-1844, 1972.

Skofronick-Jackson, G. M., Kim, M.-J., Weinman, J. A., and Chang, D.-E.: A physical model to determine snowfall over Land by microwave radiometry, IEEE Trans. Geosci. Remote Sens., 42, 1047-1058, 2004.

Teschl, F., Randeu, W. L., and Teschl, R.: Single scattering from frozen hydrometeors at microwave frequencies, Atmos. Res., doi:10.1016/j.atmosres.2009.09.001, 2009.

Warren, S. G.: Optical constants of ice from the ultraviolet to the microwave, Appl. Optics, 23, 1206-1225, 1984. 